\title{
Producto interno Bruto en América Latina y el Caribe: Relaciones entre crecimiento económico y sustentabilidad ambiental
}

\author{
Quinde-Rosales, Victor ${ }^{1}$ \\ Bucaram-Leverone, Rina ${ }^{2}$ \\ Bucaram-Leverone, Martha ${ }^{3}$ \\ Silvera-Tumbaco, Carla ${ }^{4}$
}

\section{Resumen}

Las economías en América Latina y el Caribe atraviesan por relevantes transformaciones, asociados a las capacidades del aparato productivo y a sus capacidades para revertir en la sociedad beneficios asociados al empleo, desigualdad y pobreza. El propósito de esta investigación es analizar el Producto Interno Bruto PIB en América Latina y el Caribe. Se abordan como elementos de análisis el crecimiento económico y el deterioro ambiental desde el PIB Per Cápita y el dióxido de carbono $\mathrm{CO}_{2}$ en los países de América Latina y el Caribe considerados en vías de desarrollo y el G-8 considerados como desarrollados, durante el periodo 1960 a 2011. La metodología utilizada estuvo dirigida a la realización de un razonamiento inductivo bajo un paradigma empírico-analítico, se hizo uso de la prueba de raíz unitaria Dickey-Fuller Aumentada - DFA, se generó un modelo de vectores autorregresivos - VAR, se realizó la prueba de causalidad de Granger y se desarrolló la prueba de cointegración de Johansen. Los resultados demuestran la no estacionariedad de las variables en estudio para ambos países, se obtuvo un modelo VAR de dos variables con un número de rezagos óptimo de catorce - VAR ${ }_{2}(14)$ para el caso de América Latina y el Caribe y un modelo VAR de dos

Recibido: 20.11.18 Aceptado: 20.01.19

1 Economista Agrícola con Maestría en Economía Agraria, Director del Instituto de Investigación de Economía Agrícola y Desarrollo Rural - INEAR, docente de la Facultad de Economía Agrícola de la Universidad Agraria del Ecuador. E-mails: vquinde@uagraria.edu.ec

2 Economista Agrícola con Maestría en Economía Agraria y un Doctorado en Ciencias Ambientales, Decana y docente de la Facultad de Economía Agrícola de la Universidad Agraria del Ecuador. E-mails: rbucaram@uagraria.edu.ec

3 Economista Agrícola con Maestría en Docencia Superior y un Doctorado en Ciencias Ambientales, docente de la Facultad de Economía Agrícola de la Universidad Agraria del Ecuador. E-mails: mbucaram@uagraria.edu.ec

4 Ingeniera en Sistemas Computacionales con Maestría en Currículo e Instrucción, docente de la Facultad de Economía Agrícola de la Universidad Agraria del Ecuador. Email: csilvera@ uagraria.edu.ec 
variables con un número de rezagos óptimo de cuatro - $\mathrm{VAR}_{2}(4)$ para el caso del G-8 a los cuales se les realizaron la prueba de causalidad demostrando una bidireccionalidad para América Latina y el Caribe y una unidireccionalidad por parte del PIB per cápita al $\mathrm{CO}_{2}$ para el G-8. Se concluye que ambas variables se mueven conjuntamente a lo largo del tiempo y las diferencias entre ellas son estables, para ambos casos existe al menos un vector o relación de cointegración.

Palabras clave: crecimiento económico; producto interno bruto; distribución de la renta: economía del medio ambiente.

\title{
Causality between economic growth and environmental deterioration in latin america and the caribbean
}

\begin{abstract}
Abstrac
Las economías en América Latina y el Caribe atraviesan por relevantes transformaciones, asociados a las capacidades del aparato productivo y a sus capacidades para revertir en la sociedad beneficios asociados al empleo, desigualdad y pobreza. El propósito de esta investigación es analizar el Producto Interno Bruto PIB en América Latina y el Caribe. Se abordan como elementos de análisis el crecimiento económico y el deterioro ambiental desde el PIB Per Cápita y el dióxido de carbono $\mathrm{CO}_{2}$ en los países de América Latina y el Caribe considerados en vías de desarrollo y el G-8 considerados como desarrollados, durante el periodo 1960 a 2011. La metodología utilizada estuvo dirigida a la realización de un razonamiento inductivo bajo un paradigma empírico-analítico, se hizo uso de la prueba de raíz unitaria Dickey-Fuller Aumentada - DFA, se generó un modelo de vectores autorregresivos - VAR, se realizó la prueba de causalidad de Granger y se desarrolló la prueba de cointegración de Johansen. Los resultados demuestran la no estacionariedad de las variables en estudio para ambos países, se obtuvo un modelo VAR de dos variables con un número de rezagos óptimo de catorce - $\operatorname{VAR}_{2}(14)$ para el caso de América Latina y el Caribe y un modelo VAR de dos variables con un número de rezagos óptimo de cuatro - $\operatorname{VAR}_{2}(4)$ para el caso del G-8 a los cuales se les realizaron la prueba de causalidad demostrando una bidireccionalidad para América Latina y el Caribe y una unidireccionalidad por parte del PIB per cápita al $\mathrm{CO}_{2}$ para el G-8. Se concluye que ambas variables se mueven conjuntamente a lo largo del tiempo y las diferencias entre ellas son estables, para ambos casos existe al menos un vector o relación de cointegración.
\end{abstract}

Keywords: Economic growth, Economic development, Income distribution, Environmental economics, Environmental impact assessment 


\section{Introducción}

El tema del producto interno bruto (PIB) es sumamente amplio, por los cual, es necesario establecer una delimitación en cuanto a los elementos que lo genera. Por otro lado, el término de desarrollo sustentable requiere precisión, al tomar en cuenta que hoy, los elementos que lo definen están asociado al crecimiento económico y al ambiente.

A partir de ello, se traza como propósito de esta investigación analizar el PIB en América Latina y el Caribe, abordando elementos como el crecimiento económico y el deterioro ambiental desde el PIB Per Cápita y el dióxido de carbono - $\mathrm{CO} 2$ en los países de América Latina y el Caribe, considerados en vías de desarrollo y el G-8, durante el periodo 1960 a 2011.

Desde la perspectiva metodológica, se asume un tipo de razonamiento inductivo con aplicación de pruebas econométricas para medir la probabilidad de los argumentos establecidos en función a la causalidad del $\mathrm{CO}^{5}$ y $\mathrm{PIB}$ per cápita ${ }^{6}$ de América Latina y el Caribe y el G-8. Se esboza como proceso de desarrollo de la investigación un proceso unimétodo con un paradigma empíricoanalítico (Bacon, 1949; citado por Duque, 2015) que permite reflejar la realidad de la forma más fiel y neutral posible de la investigación realizada.

Se utilizó una base de datos de serie temporales de frecuencia anual con un periodo de evaluación de 1960 al 2011 de las variables homologadas $\mathrm{CO}_{2} \mathrm{y}$
PIB per cápita de cada uno de los países en estudio, cuya base fue obtenida del CDIAC (Carbon Dioxide Information Analysis Center) y el Banco Mundial respectivamente. La idea es proponer un análisis que determine la estacionariedad de las variables mediante el uso de la prueba Dickey-Fuller Aumentada (DFA) para evitar obtener resultados espurios $^{7}$ en regresiones con series de tiempo no estacionarias, para establecer una relación entre el $\mathrm{CO}_{2}$ y el PIB per cápita se usa el modelo de Vectores Autorregresivos (VAR), entendiendo que la posible existencia de una relación entre variables puede especificar como tal un sinónimo de causalidad. Finalmente se aplicará un método de análisis de cointegración entendiendo que dos o más series de tiempo con tendencias estocásticas puede evolucionar juntas de forma tan estrecha a largo plazo que puede parecer que tienen el mismo componente tendencial; es decir, que parece que tengan una tendencia común.

\section{Desarrollo sostenible: Una conceptualización necesaria}

El término desarrollo sostenible a pesar de tener múltiples interpretaciones se encuentra omnipresente en el pensamiento y las acciones de los actores involucrados en la actividad medioambiental y de desarrollo (Urteaga, 2009); por lo que se puede afirmar bajo el criterio de Brundtland (1987), que el desarrollo sostenible requiere de un

\footnotetext{
Emisiones totales de $\mathrm{CO} 2$ de los combustibles fósiles (1000 toneladas métricas) Ingresos por persona (US \$ 2.000 fijo)

7 Espurio es la relación estadística de dos o más variables que aun así no poseen una relación de casualidad o coincidencia.
} 
sistema de producción que presente la idea de preservar el medio ambiente.

A pesar de esto, Bermejo (2014) expone el mal uso que dan los Estados al término desarrollo sostenible defendiendo el crecimiento ilimitado y proponiendo estrategias de crecimiento económico que dejan de lado la conservación de la vida social, natural y diversa. Por su parte, Beckerman (1972) describe que a pesar de que el crecimiento económico genera un deterioro en el medioambiente en la etapa inicial, a largo plazo es razonable que los países deban enriquecerse para tener políticas medioambientales adecuadas.

Esta idea de que el crecimiento continuo va en el sentido de la protección del medioambiente se desarrolla por parte de los neoclásicos desde 1970, hasta llegar a los estudios de Grossman et al, $(1993,1995)$ que con una base empírica establece una correlación entre el crecimiento económico y las evoluciones medioambientales, manifestando que las emisiones contaminantes aumentan con el incremento de la renta per cápita, antes de decrecer.

Al respecto, Medina et al, (2017), manifiestan que mencionado pensamiento asienta sus bases en relación con las investigaciones de la década de los cincuenta donde se estudiaba el crecimiento económico y la inequidad en la distribución de la renta (Ahluwalia, 1976; Alesina et al, 1994; Barro, 2000; Galindo, 2002; Álvarez, 2007; Núñez, 2016), principalmente con el aporte de Kuznets (1955), el cual entiende según Araujo et al, (2015) que crecimiento económico dista de ser la única y más precisa medida de bienestar; en una de sus investigaciones sobre la relación que presenta el crecimiento económico (medido a través del PIB per cápita ${ }^{8}$ ) y la distribución del ingreso; se postula que mencionadas variables presentan una relación en forma de u invertida. Entendiendo a esto como el aumento del ingreso a largo plazo genera una menor desigualdad (Correa et al, 2005).

Por otro lado, la teoría de desarrollo sostenible sustentada en la curva medioambiental de Kuznets explora la relación existente entre el crecimiento económico y la calidad ambiental, intentando establecer dos etapas una de corto plazo donde el crecimiento económico promueve un mayor deterioro medio ambiental, y una de largo plazo. Expone que a medida que las economías presentan mayores ingresos, su crecimiento económico beneficia al medio ambiente, estableciendo como idea que la calidad del medio ambiente mejora con el incremento en el ingreso. Mencionada evidencia se sustenta con lo encontrado en países desarrollados (Correa et al, 2005).

\section{Crecimiento económico y deterioro medioambiental: Relaciones existentes}

Con base en mencionada premisa se han realizados una serie de investigaciones que permiten tener 5 Ingreso per cápita o renta per cápita es un indicador económico que mide la relación existente
entre el nivel de renta de un país y su población. 
evidencia empírica sobre la relación planteada, en este sentido, existe un único tipo de relación entre el crecimiento económico y el deterioro medioambiental en cada uno de los países estudiados por los autores.

Así, Grossman et al, (1993), correlacionó la renta per cápita con medidas de contaminación del aire y del agua, expresando que las emisiones contaminantes aumentan con el incremento de la renta per cápita, antes de decrecer. Selden et al, (1994), corroboraron la relación entre contaminación y desarrollo económico de otros estudios evaluando emisiones de cuatro contaminantes atmosféricos importantes, partículas en suspensión, dióxido de azufre, óxidos de nitrógeno y monóxido de carbono de una base de datos de panel de varias naciones. Por su parte, Holtz-Eakin et al, (1995) mediante el uso de datos de panel global examinaron la relación entre el PIB per cápita y las emisiones de $\mathrm{CO}_{2}$, estableciendo que existe una disminución de la Propensión Marginal a Emitir - MPE $\mathrm{CO}_{2}$ a medida que aumenta el desarrollo económico; a pesar de establecer este supuesto el autor menciona que en el desarrollo de los años las emisiones de $\mathrm{CO}_{2}$ se mantendrán en $1,8 \%$ al año, valor no correspondiente con el crecimiento promedio del PIB, debido a la inferencia creada por los países de menor Propensión Marginal a Emitir-MPE, que presentan un notable incremento en su PIB y en su población.

En este orden de ideas, Stern (1996) expone como la relación entre la degradación ambiental y el ingreso per cápita ha sido usado para implicar al crecimiento económico en la eventual corrección de los impactos ambientales de las primeras etapas de desarrollo económico. Los autores generan una crítica a la relación entre la degradación ambiental y el ingreso per cápita, pues argumentan que el concepto depende de un modelo de economía del cual no existe comentario alguno de la calidad medioambiental a las posibilidades de producción, y que el comercio presenta un efecto neutral sobre la degradación medioambiental; adicionalmente generaron un econométrico con previsiones del Banco Mundial - BM al 2025, estableciendo que las emisiones de $\mathrm{SO}_{2}$ seguirán aumentando, precisando que la pérdida de bosques se estabiliza antes del final del período, a pesar de que la deforestación tropical continuará a un ritmo constante.

Otro autor importante es Ekins (1997), quien evalúa econométricamente la relación entre ciertos indicadores de calidad ambiental e ingresos; manifiesta una escasa relación presentando una relación monótonamente creciente $y$ concluye desde el punto de vista de la sostenibilidad ambiental, que la relación entre ingreso y medio ambiente sigue siendo problemática y amerita una política ambiental que relacione el crecimiento futuro de los ingresos con el desarrollo sostenible.

Por su parte, Moomaw et al, (1997) compararon dos modelos de relación entre calidad ambiental y crecimiento económico entre dieciséis países industriales evaluados; estableciendo que las emisiones de $\mathrm{CO}_{2}$ no disminuyen a mayor ingreso y menos aún de la existencia de un punto de inflexión, las emisiones de $\mathrm{CO}_{2}$ decrecientes no presentan una correlación con los niveles de ingresos sino con el tiempo entendiendo que este reacciona a choques externos.

Para Bruyn et al, (1998), al abordar la base empírica de la relación entre 
el crecimiento económico y el medio ambiente, establecen como primera hipótesis que la relación entre los ingresos y las emisiones estimadas a partir de los datos del panel no tiene por qué ser válida para países específicos a través del tiempo, para el análisis se usa como variable el $\mathrm{CO}_{2}$, $\mathrm{NOX}$ y $\mathrm{SO}_{2}$ en cuatro republicas Países Bajos, Reino Unido, Estados Unidos y Alemania Occidental; encontrando que los patrones de tiempo de estas emisiones se correlacionan positivamente con el crecimiento económico y que las reducciones de emisiones pueden haberse logrado como resultado de cambios estructurales y tecnológicos en la economía. Friedl et al, (2003) exploraron la relación entre el desarrollo económico y las emisiones de $\mathrm{CO}_{2}$ en el caso de un pequeño país abierto e industrializado como Austria; los autores encuentran una relación cubica en forma de $\mathrm{N}$ con un quiebre estructural a mediados de los años setenta justificado por el precio del petróleo.

Sheldon (2007) reevalúa las estimaciones y el análisis desarrollado por Holtz-Eakin et al, (1995) y agrega cerca de 20 años y 45 países a la muestra; el autor valida la investigación mencionada y establece que las predicciones son mayores que las estimaciones originales. Mientras que Huang et al, (2008) analizaron el consumo de energía y el PIB de 82 países, datos proporcionados por el BM los cuales fueron evaluados a través de un enfoque $\mathrm{GMM}^{9}$ para la estimación de datos de panel por el modelo VAR ${ }^{10}$; los autores establecen que no existe una relación causal entre el consumo de energía y el crecimiento económico, los países de ingresos medios sugestionan el consumo de energía positivamente, los países de ingresos altos generan un consumo de energía con tendencia negativa.

Desde otra perspectiva, $\mathrm{He}$ et al, (2010) evalúan la relación inversa del crecimiento económico y el medio ambiente manifestando que los modelos de regresión cuadrática o cúbica completamente paramétricos y utilizados tradicionalmente para el análisis no son incorrectos, pero mencionado enfoque carece de flexibilidad ya que puede no detectar la verdadera forma de la relación, para el análisis los autores utilizaron métodos de modelado paramétrico no lineal, semi-paramétrico y flexible validando su hipótesis.

A partir del desarrollo de una aplicación de la teoría del crecimiento, Dinda (2004) proporciona una explicación teórica de la relación inversa del crecimiento económico y el medio ambiente mediante el uso del teorema de sobre, entendiendo que, en un proceso de desarrollo económico, la tecnología primero se difunde, luego se vuelve regulada y finalmente es eliminada por otra nueva tecnología. Así mismo, utilizando la metodología de cointegración de umbral no lineal y un modelo VEC para el caso de Reino Unido, Fosten et al, (2012) evidencian que no existe una relación inversa del

9 Técnica econométrica genérica de estimación de parámetros de una ecuación de regresión, desarrollada como una extensión del método de los momentos.

10 Modelo de Vectores Autorregresivos, modelo de ecuaciones simultáneas formado por un sistema de ecuaciones de forma reducida sin restringir 
$\mathrm{CO}_{2}$ per cápita, emisiones de $\mathrm{SO}_{2}$ y el PIB.

En este sentido, es evidente como la literatura referenciada, manifiesta que es evidente la relación existente entre el crecimiento económico de países en vías de desarrollo y desarrollados. $\mathrm{Su}$ deterioro medio ambiental se han desarrollado estudios empíricos y en diversas pruebas estadísticas siendo aplicadas sobre datos de panel y series de tiempo (Ahmed et al, 2014); técnicas usadas con grupos de países, y países de manera individual.

\section{Producto Interno Bruto per cápita ${ }^{11}$ a lo largo del ciclo económico para el caso de América Latina y el Caribe, y el G-8}

En esta sección se pretende establecer si la emisión total de $\mathrm{CO}_{2}{ }^{12}$ (Dióxido de Carbono) presenta una relación de cointegración con el PIB (Producto Interno Bruto) per cápita ${ }^{13}$ a lo largo del ciclo económico para el caso de América Latina y el Caribe, y el G-8 ${ }^{14}$. Entendiendo que estos convergen en una tendencia común en el largo plazo en ambos grupos de países. En este sentido, desde una perspectiva empírica, se busca presentar nuevos aportes frente a la disyuntiva existente entre crecimiento económico y el medio ambiente en países en vías de desarrollo y países desarrollados.

El diseño de la investigación plasmado en el documento busca caracterizar la relación de causalidad existente entre el crecimiento económico $y$ el deterioro medio ambiental entre países desarrollados y en vías de desarrollo, bajo la teoría del desarrollo sostenible mediante el análisis de autores que han desarrollado estudios similares, para luego establecer el marco metodológico que englobe el desarrollo del estudio empírico. Posterior a ello, se presentará el modelo de vectores autorregresivos que evidencie la simultaneidad de los índices antes mencionados sumado al test de Granger el cual establece la causalidad existente y finalmente la prueba de Johansen para determinar la existencia de cointegración entre las variables.

La relación entre el Dióxido de Carbono $\left(\mathrm{CO}_{2}\right)$ y el Producto Interno Bruto (PIB) Per-cápita, para el caso de América Latina y el Caribe presenta una pendiente positiva, mientras que en el caso del G-8 mencionada relación presenta una tendencia irregular la cual tiene puntos críticos altos en 1970 y 1992, y puntos críticos bajos en 1982 y 2009 , presentando en su curva una forma de "N"; comportamiento que concuerda con los aportes empíricos de Tarazona (1999) (Figura 1).

11 PIB per-cápita en dólares constantes del 2000. Se ha tenido en cuenta la inflación, pero no las diferencias en el costo de la vida entre los países.

12 Cantidad total de $\mathrm{CO}_{2}$ en 1000 toneladas métricas emitidas por el consumo de todo tipo de combustibles fósiles.

13 PIB per-cápita en dólares constantes del 2000. Se ha tenido en cuenta la inflación, pero no las diferencias en el costo de la vida entre los países.

14 Se denomina con el numerónimo G8, al grupo de los ocho, un grupo de países con las economías más industrializadas del planeta. Está conformado por Rusia, Canadá, Estados Unidos, Francia, Italia, Alemania, Reino Unido y Japón 
Figura $1 \mathrm{~A}$.

\section{Relación entre CO2 Per-cápita y el PIB Per-cápita. América Latina} y El Caribe

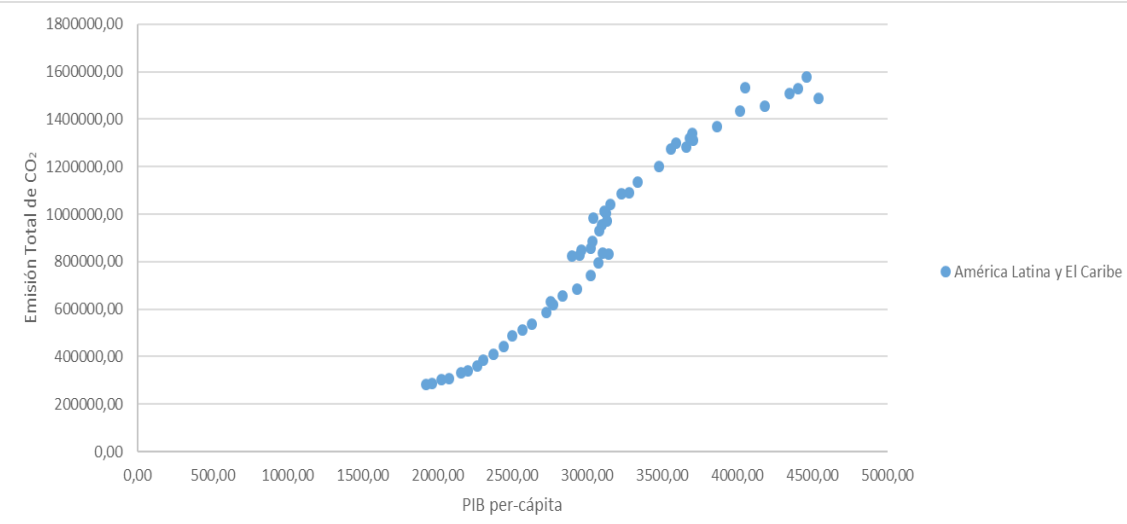

Fuente:

Figura 1B

Relación entre CO2 Per-cápita y el PIB Per-cápita. G-8

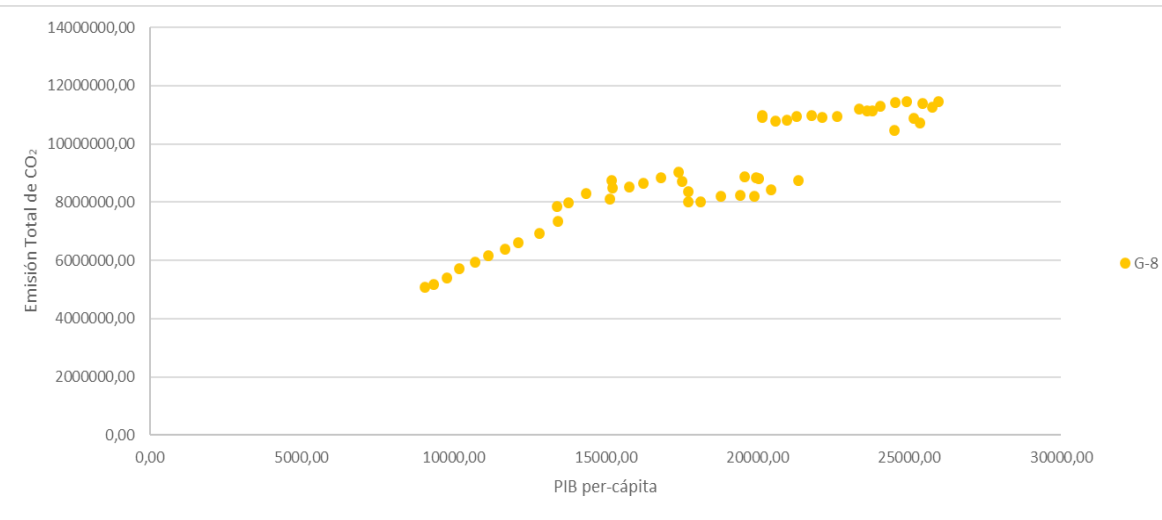

Fuente:

Para determinar el criterio de tendencia determinística o estocástica se realizó la prueba de Dickey-Fuller Aumentada (DFA). Los resultados de esta prueba muestran que en ambos grupos los indicadores aprueban la hipótesis nula, indicando que tanto el $\mathrm{CO}_{2}$ como el PIB Per-cápita son series que tienen raíz unitaria siendo estas no estacionarias (Tabla 1). 
pp. 769-784

Revista Venezolana de Gerencia, Año 24 N 87, 2019

\section{Tabla 1.}

Prueba de Raíz Unitaria $\mathrm{CO}_{2}$ y PIB Per-cápita

\begin{tabular}{ccccc}
\hline \multirow{2}{*}{ Grupo } & Variable & \multicolumn{2}{c}{ Prueba DFA } & \multirow{2}{*}{ Prob* $^{*}$} \\
\cline { 3 - 4 } & & $\begin{array}{c}\text { Test critical values: 5\% } \\
\text { level }\end{array}$ & t-Statistic & \\
\hline $\begin{array}{c}\text { América } \\
\text { Latina y el } \\
\text { Caribe }\end{array}$ & $\mathbf{C O}_{2}$ & -2.919952 & -0.094370 & 0.9443 \\
& PIB Per-cápita & -2.919952 & -1.080856 & 0.7166 \\
G-8 & CO $_{2}$ & -2.919952 & -1.974740 & 0.2968 \\
& PIB Per-cápita & -2.919952 & -1.734473 & 0.4082 \\
\hline
\end{tabular}

*MacKinnon (1996) one-sided p-values

Fuente: elaboración propia

Para el proceso de estimación del vector autorregresivo (VAR) es necesario establecer la longitud máxima de rezago sobre el cual se determine la relación de las variables $\mathrm{CO}_{2}$ y PIB Percápita entendiendo este criterio como el rezago óptimo. Para ello se utilizará la prueba de razón de verosimilitud, los criterios de Error de Predicción Final y los estadísticos de Akaike, Schwarz y Hannan-Quinn. Estos criterios presentan una discrepancia al momento de establecer el rezago; al momento de establecer como rezago optimo en el caso de América Latina y el Caribe este varía entre los rezagos 2, 3, 9 y 14, mientras que en el G-8 varía entre los rezagos 1 y 4 (Tabla 2).

Tabla 2A.

Criterios de Selección del Orden de Retrasos - VAR $\mathrm{CO}_{2}$ PIB Per-cápita. América Latina y el Caribe

\begin{tabular}{|c|c|c|c|c|c|c|}
\hline Lag & LogL & LR & FPE & AIC & SC & HQ \\
\hline 0 & -777.5223 & NA & $2.25 e+15$ & 41.02749 & 41.11368 & 41.05815 \\
\hline 1 & -675.2612 & 188.3756 & $1.28 e+13$ & 35.85585 & 36.11442 & 35.94785 \\
\hline 2 & -662.5528 & 22.07257 & $8.12 e+12$ & 35.39751 & $35.82846^{*}$ & 35.55084 \\
\hline 3 & -657.0589 & 8.963709 & $7.54 \mathrm{e}+12^{*}$ & 35.31889 & 35.92221 & $35.53355^{*}$ \\
\hline 4 & -655.7197 & 2.044067 & $8.76 e+12$ & 35.45893 & 36.23463 & 35.73492 \\
\hline 5 & -651.8561 & 5.490299 & $8.97 e+12$ & 35.46611 & 36.41419 & 35.80343 \\
\hline 6 & -651.8186 & 0.049396 & $1.13 e+13$ & 35.67466 & 36.79512 & 36.07331 \\
\hline 7 & -650.3586 & 1.767334 & $1.34 \mathrm{e}+13$ & 35.80835 & 37.10118 & 36.26833 \\
\hline 8 & -648.4587 & 2.099844 & $1.56 e+13$ & 35.91888 & 37.38409 & 36.44019 \\
\hline 9 & -638.3809 & $10.07781^{*}$ & $1.21 \mathrm{e}+13$ & 35.59900 & 37.23658 & 36.18164 \\
\hline 10 & -635.4088 & 2.659311 & $1.38 e+13$ & 35.65309 & 37.46306 & 36.29706 \\
\hline
\end{tabular}


Producto interno Bruto en América Latina y el Caribe:

Relaciones entre crecimiento económico y sustentabilidad ambiental

Victor, Quinde-Rosales; Rina, Bucaram-Leverone; Martha, Bucaram-Leverone y

Carla, Silvera-Tumbaco

\begin{tabular}{rrrrrrr}
\hline 11 & -628.4772 & 5.472289 & $1.32 \mathrm{e}+13$ & 35.49880 & 37.48114 & 36.20410 \\
12 & -619.2194 & 6.334256 & $1.15 \mathrm{e}+13$ & 35.22208 & 37.37679 & 35.98871 \\
13 & -610.5310 & 5.030151 & $1.08 \mathrm{e}+13$ & 34.97532 & 37.30241 & 35.80328 \\
14 & -602.6341 & 3.740617 & $1.13 \mathrm{e}+13$ & $\mathbf{3 4 . 7 7 0 2 2}$ & 37.26969 & 35.65951
\end{tabular}

* indicates lag order selected by the criterion

LR: sequential modified LR test statistic (each test at $5 \%$ level)

FPE: Final prediction error

AIC: Akaike information criterion

SC: Schwarz information criterion

$H Q$ : Hannan-Quinn information criterion

Fuente: elaboración propia

Tabla 2B.

Criterios de Selección del Orden de Retrasos - VAR $\mathrm{CO}_{2}$ PIB Per-cápita. G-8

\begin{tabular}{|c|c|c|c|c|c|c|}
\hline Lag & LogL & LR & FPE & AIC & Sc & HQ \\
\hline 0 & -927.3869 & NA & $6.01 \mathrm{e}+18$ & 48.91510 & 49.00129 & 48.94577 \\
\hline 1 & -830.3488 & 178.7545 & $4.49 \mathrm{e}+16$ & 44.01836 & $44.27692^{*}$ & 44.11035 \\
\hline 2 & -829.5215 & 1.436884 & $5.32 \mathrm{e}+16$ & 44.18534 & 44.61628 & 44.33867 \\
\hline 3 & -828.8382 & 1.114781 & $6.37 e+16$ & 44.35991 & 44.96323 & 44.57456 \\
\hline 4 & -814.7577 & $21.49135^{*}$ & $3.78 e+16^{*}$ & $43.82935^{*}$ & 44.60505 & $44.10534^{*}$ \\
\hline 5 & -813.2488 & 2.144158 & $4.38 e+16$ & 43.96046 & 44.90854 & 44.29778 \\
\hline 6 & -809.6564 & 4.726853 & $4.58 e+16$ & 43.98192 & 45.10237 & 44.38057 \\
\hline 7 & -805.5206 & 5.006483 & $4.70 \mathrm{e}+16$ & 43.97477 & 45.26760 & 44.43475 \\
\hline 8 & -804.8922 & 0.694557 & $5.88 e+16$ & 44.15222 & 45.61743 & 44.67353 \\
\hline 9 & -802.1583 & 2.733869 & $6.68 e+16$ & 44.21886 & 45.85645 & 44.80150 \\
\hline 10 & -800.1937 & 1.757878 & $8.06 e+16$ & 44.32598 & 46.13595 & 44.96995 \\
\hline 11 & -797.2699 & 2.308215 & $9.49 e+16$ & 44.38263 & 46.36497 & 45.08793 \\
\hline 12 & -794.2014 & 2.099541 & $1.15 e+17$ & 44.43165 & 46.58637 & 45.19828 \\
\hline 13 & -792.7262 & 0.854026 & $1.58 \mathrm{e}+17$ & 44.56454 & 46.89163 & 45.39250 \\
\hline 14 & -784.3152 & 3.984176 & $1.61 \mathrm{e}+17$ & 44.33238 & 46.83185 & 45.22167 \\
\hline $\begin{array}{l}\text { EPE: } \\
\text { AIC: A } \\
\text { SC: S } \\
\text { HQ: H } \\
\text { Euent }\end{array}$ & $\begin{array}{l}\text { lag orde } \\
\text { l predial mo } \\
\text { re inform } \\
\text { arz inforr } \\
\text { an-Quinr } \\
\text { aboració }\end{array}$ & $\begin{array}{l}\text { NA } \\
\text { elected by } \\
\text { d LR test } \\
\text { error } \\
\text { an criterion } \\
\text { ion criterio } \\
\text { formation a } \\
\text { ropia }\end{array}$ & $\begin{array}{l}\text { criterion } \\
\text { stic (each } \\
\text { ion }\end{array}$ & 50 & & \\
\hline
\end{tabular}


Para el desarrollo del presente documento se destaca cómo modelo adecuado el rezago establecido por el criterio de información de Akaike que se presenta en el décimo cuarto rezago para el caso de América Latina y el Caribe, y en el cuarto rezago para el G-8. Entendiendo que el rezago optimo del VAR entre el $\mathrm{CO}_{2}$ y el PIB Per-cápita es de catorce rezagos - $\operatorname{VAR}_{2}(14)$ para el caso de América Latina y el Caribe y el rezago óptimo del VAR entre el $\mathrm{CO} 2$ y el PIB Per-cápita es de cuatro rezagos - $\operatorname{VAR}_{2}(4)$ para el caso del G-8, se determina que los indicadores presentan respuesta a los shocks entre variables y entre sí mismas propagándose en el largo plazo.
Para establecer la causalidad se realizó una prueba de Granger cuyos resultados muestran en el caso de América Latina y el Caribe una causalidad bidireccional rechazando las hipótesis nulas que el PIB Per-cápita no causa en el sentido de Granger al $\mathrm{CO}_{2}$ y que el $\mathrm{CO}_{2}$ no causa en el sentido de Granger al PIB Per-cápita. Para el caso del G-8 esta muestra una unidireccionalidad rechazando la hipótesis nula que el PIB Per-cápita no causa en el sentido de Granger al $\mathrm{CO}_{2}$ y aprobando la hipótesis nula que el $\mathrm{CO}_{2}$ no causa en el sentido de Granger al PIB Per-cápita (Tabla 3). Esto se evidencia al observar los valores de los $p$ values en la tabla

\section{Tabla 3.}

\section{Prueba de Causalidad de Granger - VAR $\mathrm{CO}_{2}$ y PIB Per-cápita}

\begin{tabular}{ccccc}
\hline \multirow{2}{*}{ Grupo } & \multirow{2}{*}{ Hipótesis nula } & Chi-sq & Df & Prob. \\
\cline { 3 - 5 } & & 35.92645 & 14 & 0.0011 \\
\hline \multirow{2}{*}{$\begin{array}{c}\text { América Latina y } \\
\text { el Caribe }\end{array}$} & $\mathrm{CO}_{2}$ no causa a PIB Per-cápita & 14 & 0.0219 \\
& $\mathrm{PIB} \mathrm{Per-cápita} \mathrm{no} \mathrm{causa} \mathrm{a} \mathrm{CO}_{2}$ & 26.56334 & 4 & 0.5826 \\
\multirow{2}{*}{ G-8 } & $\mathrm{CO}_{2}$ no causa a PIB Per-cápita & 2.853532 & 4 & 0.0000 \\
\hline
\end{tabular}

Fuente: elaboración propia

La tabla de diálogo resumen indica las cuatro especificaciones de Cointegración tanto en la prueba de la Traza como en la del Maximun Eigenvalue, siendo estas la no tendencia determinística en los datos, la de tendencia determinística lineal en los datos y la tendencia determinística cuadrática en los datos, para el caso de estudio se seleccionará sólo intercepto en la ecuación de cointegración (CE) y no tendencia en el VAR para la de tendencia determinística lineal en los datos en ambos casos (Tabla IV). 
Tabla 4.

Test de Cointegración de Johansen - Resumen de Supuestos

\begin{tabular}{ccccccc}
\hline Grupo & $\begin{array}{c}\text { Data } \\
\text { Trend: }\end{array}$ & None & None & Linear & Linear & Quadratic \\
\hline $\begin{array}{c}\text { América } \\
\text { Latina y el } \\
\text { Caribe }\end{array}$ & Test Type & $\begin{array}{c}\text { No Intercept } \\
\text { No Trend }\end{array}$ & $\begin{array}{c}\text { Intercept No } \\
\text { Trend }\end{array}$ & $\begin{array}{c}\text { Intercept } \\
\text { No Trend }\end{array}$ & $\begin{array}{c}\text { Intercept } \\
\text { Trend }\end{array}$ & $\begin{array}{c}\text { Intercept } \\
\text { Trend }\end{array}$ \\
& Trace & 1 & 2 & 2 & 2 & 2 \\
\hline \multirow{2}{*}{ G-8 } & Max-Eig & 1 & 2 & 2 & 2 & 2 \\
& Test Type & No Intercept & Intercept No & Intercept & Intercept & Intercept \\
& Trace & 1 & Trend & No Trend & Trend & Trend \\
& Max-Eig & 1 & 1 & 2 & 0 & 0 \\
\hline
\end{tabular}

Selected $\left(0.05\right.$ level $\left.^{*}\right)$ Number of Cointegrating Relations by Model

${ }^{*}$ Critical values based on MacKinnon-Haug-Michelis (1999)

Fuente: elaboración propia

El test de Johansen presenta como criterio con un nivel del $5 \%$ de confianza se rechaza la hipótesis nula de no cointegración en favor de una relación de cointegración y de la prueba de Máximun EigenValue concluyendo que existe al menos un solo vector o relación de cointegración para el caso de América Latina y el Caribe, y el G-8 (Tabla V).

\section{Tabla 5A}

\section{Test de Cointegración de Johansen - $\mathrm{CO}_{2}$ y PIB Per-cápita de 14 Rezagos. América Latina y el Caribe}

\begin{tabular}{|c|c|c|c|c|}
\hline \multirow{2}{*}{$\begin{array}{c}\text { Número de ecuaciones } \\
\text { de cointegración bajo } \\
\text { Ho }\end{array}$} & \multicolumn{4}{|c|}{ Prueba de Rango de Cointegración Sin Restricciones de Traza } \\
\hline & Valor Propio & $\begin{array}{l}\text { Estadístico de la } \\
\text { Traza }\end{array}$ & $\begin{array}{l}\text { Valor critico } \\
\quad 0,05\end{array}$ & P-valor* \\
\hline Ninguna & 0.283473 & 13.11502 & 12.32090 & 0.0368 \\
\hline Cuando más 1 & 0.020899 & 0.781476 & 4.129906 & 0.4336 \\
\hline \multirow{2}{*}{$\begin{array}{c}\text { Número de ecuaciones } \\
\text { de cointegración bajo } \\
\text { Ho }\end{array}$} & \multicolumn{4}{|c|}{$\begin{array}{l}\text { Prueba de Rango de Cointegración Sin Restricciones del Máximo } \\
\text { de Valor Propio }\end{array}$} \\
\hline & Valor Propio & $\begin{array}{l}\text { Estadístico del } \\
\text { Máximo de Valor } \\
\text { Propio }\end{array}$ & $\begin{array}{c}\text { Valor critico } \\
0,05\end{array}$ & P-valor* \\
\hline Ninguna & 0.283473 & 12.33355 & 11.22480 & 0.0318 \\
\hline Cuando más 1 & 0.020899 & 0.781476 & 4.129906 & 0.4336 \\
\hline
\end{tabular}


pp. 769-784

Revista Venezolana de Gerencia, Año 24 N 87, 2019

Tabla 5B

Test de Cointegración de Johansen - $\mathrm{CO}_{2}$ y PIB

Per-cápita de 4 Rezagos. G-8

\begin{tabular}{|c|c|c|c|c|}
\hline \multirow{2}{*}{$\begin{array}{l}\text { Número de ecua- } \\
\text { ciones de cointe- } \\
\text { gración bajo Ho }\end{array}$} & \multicolumn{4}{|c|}{$\begin{array}{c}\text { Prueba de Rango de Cointegración Sin Restricciones de } \\
\text { Traza }\end{array}$} \\
\hline & Valor Propio & $\begin{array}{l}\text { Estadístico } \\
\text { de la Traza }\end{array}$ & $\begin{array}{l}\text { Valor critico } \\
\quad 0,05\end{array}$ & P-valor* \\
\hline Ninguna & 0.235133 & 12.97670 & 12.32090 & 0.0388 \\
\hline A lo más 1 & 0.008014 & 0.378198 & 4.129906 & 0.6017 \\
\hline \multirow[b]{2}{*}{$\begin{array}{l}\text { Número de ecua- } \\
\text { ciones de cointe- } \\
\text { gración bajo Ho }\end{array}$} & \multicolumn{4}{|c|}{$\begin{array}{c}\text { Prueba de Rango de Cointegración Sin Restricciones del } \\
\text { Máximo de Valor Propio }\end{array}$} \\
\hline & Valor Propio & $\begin{array}{c}\text { Estadístico } \\
\text { del Máximo } \\
\text { de Valor Pro- } \\
\text { pio }\end{array}$ & $\begin{array}{l}\text { Valor critico } \\
\quad 0,05\end{array}$ & P-valor* \\
\hline Ninguna & 0.235133 & 12.59851 & 11.22480 & 0.0285 \\
\hline A lo más 1 & 0.008014 & 0.378198 & 4.129906 & 0.6017 \\
\hline
\end{tabular}

*MacKinnon-Haug-Michelis (1999) p-values

Fuente: elaboración propia

Al estudiar la relación entre el $\mathrm{CO}_{2}$ y el PIB Per-cápita para el caso de América Latina y el Caribe se determinó que la relación de ambos indicadores es de tendencia positiva, no estacionaria. Por lo que fue necesario evaluar su relación bajo la prueba de Johansen, de acuerdo con el test de cointegración, el rango de cointegración de las variables analizadas es $r=1$ bajo la especificación de intercepto en la ecuación de cointegración, y tendencia determinística lineal en los datos con 14 rezagos en los términos VAR en diferencias. Bajo el criterio de causalidad se determinó que según la prueba de Granger el modelo presenta bidireccionalidad en términos de causalidad de Granger del PIB Per Cápita al $\mathrm{CO}_{2}$ y viceversa.

Para el caso del G-8, se determinó que la relación de ambos indicadores es de tendencia irregular, no estacionaria.
Por lo que fue necesario evaluar su relación bajo la prueba de Johansen, de acuerdo con el test de cointegración, el rango de cointegración de las variables analizadas es $r=1$ bajo la especificación de intercepto en la ecuación de cointegración, y tendencia determinística lineal en los datos con 4 rezagos en los términos VAR en diferencias. Bajo el criterio de causalidad se determinó que según la prueba de Granger el modelo presenta unidireccionalidad en términos de causalidad de Granger del PIB Per Cápita al $\mathrm{CO}_{2}$.

\section{Reflexiones finales}

El estudio demuestra que existe una relación empírica entre los niveles de contaminación ambiental de los países evaluados y su alcance en términos de desarrollo económico. América Latina y 
el Caribe presentan una relación entre el $\mathrm{CO}_{2}$ Per-cápita y el PIB Per-cápita con tendencia positiva, mientras que el G-8 presenta una curva en forma de "N" con irregularidades, según la premisa establecida en la curva ambiental de Kuznets.

Ante la diversidad de respuestas encontradas en los estudios revisados, se torna necesario generar nuevas investigaciones, en las cuales se apliquen metodologías diversas metodologías para el fortalecimiento de los resultados existentes en esta temática.

Establecer la relación entre el producto interno bruto per cápita (PIB Per Cápita) y las emisiones totales de Dióxido de Carbono $\left(\mathrm{CO}_{2}\right)$ representa un interés para establecer políticas públicas que reorienten la emisión de $\mathrm{CO}_{2}$ y el desarrollo de tecnologías limpias.

\section{Referencias Bibliográficas}

Ahluwalia, Montek (1976), Inequality, poverty and development, Journal of Development Economics, No. 3, pp. 307-342.

Ahmed, Khalid; Shahbaz, Muhammad; Qasing, Ahmer y Long, Wei (2014), The linkages between deforestation, energy and growth for environmental degradation in Pakistan. Ecological Indicators, No. 49, pp. $95-103$.

Alesina, Alberro. y Rodrik, Dany (1994), Distributive politics and economic growth, Quarterly Journal of Economics, Vol 109, No. 2, pp. 465-490.

Álvarez, Agustín (2007), Distribución de la renta y crecimiento económico, Información Comercial Española, ICE: Revista de economía, No. 835, (Ejemplar dedicado a: Nuevas tendencias en política fiscal), pp. 95-10

Araujo A., Jair y Cabral, Janaina (2015), Relación entre la desigualdad de la renta y el crecimiento económico en Brasil: 1995-2012. Problemas del desarrollo, Vol 46, No. 180, pp. 129150.

Bacon, Francis (1949), Novum Organum. Buenos Aires: Losada. (Versión Original 1620)

Barro, Robert (2000), Inequality and growth in a panel of countries, Journal of Economic Growth, Vol 5, No. 1, pp. 5-32.

Beckerman, Wilfred (1972), Economists, scientists, and environmental catastrophe, Oxford Economic Papers, Vol. 24, No. 3.

Bermejo, Roberto (2014), Del desarrollo sostenible según Brundtland a la sostenibilidad como biomimesis, Instituto de Estudios sobre Desarrollo y Cooperación Internacional, ISBN: 978-84-89916-92-0.

Brundtland, G. (1987), Report of the World Commission on Environment and Development - Our Common Future, Organización de las Naciones Unidas. Recuperado de: https://web.archive.org/ web/20111201061947/http://worldinbalance.net/pdf/1987-brundtland.pdf.

Bruyn, S. M., Van Den Bergh, J. C. J. M. y Opschoor, J. B. (1998), Economic growth and emissions: reconsidering the empirical basis of environmental Kuznets curves, Ecological Economics, pp. 161-175.

Correa, Francisco, Vasco, Andrés y Pérez, Catalina (2005), La Curva Medioambiental de Kuznets: Evidencia Empírica para Colombia Grupo de Economía Ambiental (GEA), Semestre Económico, Vol. 8, No. 15, pp. $13-30$

Dinda, Soumyananda (2004), Environmental Kuznets Curve Hypothesis: A Survey, Ecological Economics, pp. 
431-455.

Duque, E. (2015), Seminario Metodología de la Investigación, Universidad Nacional de Colombia

Ekins, Paul (1997), The Kuznets curve for the environment and economic growth: examining the evidence, Environment and Planning A, Vol. 29, pp. 805-830.

Fosten, Jack; Morley, Bruce; y Taylor, Tim (2012), Dynamic misspecification in the environmental Kuznets curve: Evidence from $\mathrm{CO} 2$ and $\mathrm{SO} 2$ emissions in the United Kingdom, Ecological Economics, pp. 25-33.

Friedl, Birgit y Getzner, Michael (2003), Determinants of $\mathrm{CO} 2$ Emissions in a small open Economy, Ecological Economics, pp. 133-148.

Galindo, M. A. (2002), Distribución de la renta y crecimiento económico, Anuario jurídico y económico escurialense, No. 35, pp. 473-502

Grossman, Gene y Krueger, Alan (1995), Economic Growth and the Environment, The Quarterly Journal of Economics, Vol. 110, No. 2, pp. 353377.

Grossman, Gene y Krueger, Alan B. (1993). Environmental impacts of North american free trade agreement, In: P. Garber (editor), The U.S. - Mexico Free Trade Agreement, Cambridge, MIT press, pp. 13-56

He, Jie; y Richard, Patrick (2010), Environmental Kuznets curve for $\mathrm{CO} 2$ in Canada, Ecological Economics, pp. 1083-1093.

Holtz-Eakin, Douglas y Selden, Thomas (1995), Stoking the fires? CO2 emissions and economic growth, Journal of Public Economics, pp. 85-101.

Huang, B., Hwang, M. y Yang, C. (2008), Causal relationship between energy consumption and GDP growth revisited: A dynamic panel data approach, Ecological Economics, pp. 41-54.

Kuznets, Simon, (1955), "Economic Growth and Income Inequality", American Economic Review, No. 45, pp. 1-28.

Medina, Jorge y Ayaviri, Victor (2017), Ingreso y desigualdad: la Hipótesis de Kuznets en el caso boliviano, Espacios, Vol. 38 , No. 31 , pp. 23 , ISSN 07981015.

Moomaw, William y Unruh, Gregory (1997), Are Environmental Kuznets Curves Misleading us?, Fletcher School of Law \& Diplomacy. Tufts University.

Núñez, Juan Carlos (2016), Crecimiento económico y distribución del ingreso: una perspectiva del Paraguay, Población y Desarrollo, ILPES, No. 43, pp. 54-61

Selden, Thomás M. y Song, Daqing (1994). Environmental Quality and Development: Is There a Kuznets Curve for Air Pollution Emissions?, Journal of Environmental Economics and Management, Elsevier, vol. 27(2), pp. 147-162.

Sheldon, Tamara (2007), Carbon emissions and economic growth: A replication and extension, Energy Economics.

Stern, David (1996), Progress on the Environmental Kuznets Curve?, Ecological Economics.

Stern, David; Common, Michael y Barbier, Edward (1996), Economic Growth and Environmental Degradation: The Environmental Kuznets Curve and Sustainable Development, World Development, Vol. 24, No. 7, pp. 1151-1160.

Tarazona, Marcela (1999), "El cambio climático en el desarrollo económico: 
Producto interno Bruto en América Latina y el Caribe:

Relaciones entre crecimiento económico y sustentabilidad ambiental

Victor, Quinde-Rosales; Rina, Bucaram-Leverone; Martha, Bucaram-Leverone y

Carla, Silvera-Tumbaco

revisión de la hipótesis de Kuznets", Desarrollo y Sociedad, No. 4

Urteaga, Eguzki (2009), Las teorías económicas del desarrollo sostenible, Cuadernos de Economía. Vol. 32, No. 89 , pp. 113-162. 